\title{
Delayed Recovery of Contrast Induced Kidney Injury Post Angiography: Rate and Risk Factors
}

\author{
Hilal B. Al-Saffar* \\ Adel J. Yassin** \\ Ala Ali $* * *$
}

\author{
CABM, FRCP,(Lond.,Edin), FACC \\ Dm, Dm (int.cardiology) \\ FIBMS(int.med.), FIBMS(nephro)
}

\begin{abstract}
:
Background: Exposure to Contrast media is the third leading cause of hospital acquired acute kidney injury. It follows a predictable time of onset and a less predictable scenario in recovery. This is related to certain factors, but at the end there will be asubstantial association with increased mortality, morbidity, and length of hospitalization.

J Fac Med Baghdad 2015; Vol.57, No.3 Received May. 2015 Accepted Jun.2015

Objectives: To define the Incidence of persistent contrast induced renal impairment a month after angiography and to define the risk factors for such persistence

Patients and Methods: One hundred and one patients (101) were enrolled in this study. All were referred to the Iraqi Center for Heart Diseases in Baghdad/ Iraq for coronary and/or peripheral vascular angiography from October 2009 to July 2010. All patients' clinical and laboratory data including baseline renal function tests were ordered and recorded with subsequent risk categorization. Post procedure serum creatinine checked on regular intervals ( 48 hours, one week, and then weekly for three months for those with serum creatinine consistent with the contrast induced - acute kidney injury (CI-AKI) definition at 48 hours.

Results: The mean age for the study group was $62 \pm 16.9$ with $78(77 \%)$ male. Twenty three patients $(22.7 \%)$ had diabetes mellitus. Thirteen patients $(12.7 \%)$ had a pre-existing renal impairment. Twenty one patients $(20.7 \%)$ received extradoses of contrast. Thirty two patients $(31.6 \%)$ developed CIN by the definition within 48 hours of the procedure with the mean serum creatinine of $1.8 \mathrm{mg} / \mathrm{dl}$. Seven patients $(6.8 \%)$ continued to have impaired renal function at week 4 and persisted to have such low GFR up to three months of the procedure. Baseline low GFR, Diabetes mellitus and extradoses of contrast were independent risk factors for the occurrence of CIN and delayed renal recovery.

Conclusion: The risk of poor renal recovery after contrast cannot be overlooked. Preexisting renal function impairment, diabetes mellitus, and extra doses of contrast media are independent risk factors for such delay.

Key Words: Contrast media, Nephrotoxicity, AKI, Angiography.
\end{abstract}

\section{Introduction:}

Contrast-induced nephropathy (CIN) is the third most common cause of hospital acquired renal failure, after decreased renal perfusion and nephrotoxic medications. ${ }^{1}$ It is usually defined as an impairment of the renal function occurring within 48 hours after the administration of contrast media. It is manifested as an absolute increase in serum creatinine (Scr) level by at least $44 \mathrm{mmol}$, or by relative increase of at least $25 \%$ over the base line value after excluding other causes of renal function deterioration. It should be recognized that in minority of cases, the peak increase of SCr may occur up to 5 days after contrast exposure. ${ }^{2}$ It is not surprising that the reported incidence of contrast induced- acute kidney injury (CI-AKI) varies widely across the literatures, depending on the definitions used, the

\footnotetext{
*, Dept. of Medicine/ Collage of Medicine/ Baghdad University, Baghdad/ Iraq.

**Interventional Cardiologist, Iraqi centre of heart diseases, Baghdad/ Iraq. ***Nephrologist, Renal disease and Transplantation Centre, The Medical City, Baghdad/Iraq

Email: crescent1957@yahoo.com
}

patient population, and the baseline risk factors. ${ }^{3}$ Depending on the threshold criterion for CI- AKI chosen, this variation can lead to rates of $6-35 \%$.It is accepted that, in patients with normal renal function - even in the presence of diabetes-the risk for CI-AKI is low (1-2\%).However, the incidence may be as high as $25 \%$ in patients with pre-existing renal impairment or in presence of certain risk factors, such as the combination of CKD and diabetes, CHF, advanced age, and concurrent administrationof nephrotoxic drugs. ${ }^{4,5}$ The pathogenesis of CIN is not clear. Studies in vitro and in animals suggest that CIN is due to a combination of toxic and ischemic injury to the renal tubular cells. Proximal and distal tubular injury occurs at the moment of contact with contrast medium and is thought to be due to interplay of intra-renal vasoconstriction, medullary hypoxia, and direct tubular cell death. ${ }^{6,7}$ Baseline kidney function strongly determines the risk of CIN, and the degree of Kidney impairment at baseline correlates with a proportionate risk for CIN.Diabetes, increased age, higher dose of contrast agent used, route of contrast administration (intra-arterial vs 
intravenous), congestive heart failure (CHF), hypertension, peri-proceduralshock, baselineanemia, post proceduraldrop in hematocrit, use of nephrotoxins, and nonsteroidal antiinflammatory medications, volume depletion, increased CKMB, and need for cardiac surgery after contrast exposure, have been associated with increased risk of CIN. Mehran et al devised an algorithm for risk prediction of CIN and the need for dialysis in a general population of patients who underwent coronary angiography, and subsequently validated the prediction score in a separate cohort of patients, $26 \%$ of whom had CKD (estimated glomerular filtration rate [eGFR] $<60 \mathrm{~mL} / \mathrm{min}){ }^{8}{ }^{89}$ Searching pub med yielded little about the concept of delayed renal recovery but some studies looked for mortality, long term adverse events like ESRD. Regardless of the definition of CIN and after adjustment of baseline risk factors, adverse events like ESRD was twice high in those developed CIN after exposure. ${ }^{10}$ Patients who develop CIN have been shown to have a higher rate of in-hospital mortality, diminished long-term survival when compared with those who do not. ${ }^{11,12}$ The trial reported by Sadeghi et al. found that therelative risk of 1-year mortality of 7.4 for thosewho developed CIN compared with those who did not. ${ }^{13}$ In patients with pre-existing $\mathrm{CKD}$, the impact on survival is particularly more negative. ${ }^{14}$ In a study by Wi J et al Mehran risk score predicted CIN with persistent renal dysfunction and long-term clinical outcomes in patients with AMI. ${ }^{15}$

Aim of the study : to study the rate of delay in renal recovery a month of exposure to contrast media in angiographic studies, and to identify the risk factors for such delay, this study was conducted.

\section{Patients and Methods:}

A single center prospective study recruited one hundred and one patients who had been referred to the Iraqi Center for Heart Diseases in Baghdad/ Iraq from October2006 to July 2007 for coronary or peripheral diagnostic and/or interventional angiography.After having their formal acceptance to be enrolled in this study, all patients had been interviewed,clinically examined and sendingfor routine laboratory investigations. The aims were to search for the presence of any recognized risk factors for CIN. Baseline renal function was assessed by measuring blood urea nitrogen (BUN) and serum creatinine(S. $\mathrm{Cr}$ ).Four variables MDRD equation was used for calculating eGFR as follows; eGFR $=186 \times$ Serum Creatinine ${ }^{-1.154} \mathrm{X} \mathrm{Age}^{-}$ ${ }^{0.203} \times$ [1.210 if Black] X [0.742 if Female], Serum Creatinine is in $\mathrm{mg} / \mathrm{dl}$. A periprocedural hydration with intravenous administration of $0.9 \%$ isotonic saline administered in a dose of $1-3 \mathrm{ml} / \mathrm{kg} / \mathrm{min}$ for $6-12$ hours according to patients volume status. All nephrotoxic medications had been omitted.Any patients with diabetes mellituswho was already on metformine advised to stop it and insulin therapy was introduced instead. ACEIs and diuretics were switched to other anti-hypertensive medications priorto the procedure. The patients risk category identified according to CI-AKI risk-scoring model for percutaneous coronary intervention by Mehran et al. , Table(1)

Table(1):CI-AKI risk-scoring model for percutaneous coronary intervention by Mehran et al

\begin{tabular}{|lc|}
\hline Risk factors & Integer score (calculate) \\
\hline Hypotension & 5 \\
IABP & 5 \\
CHF & 5 \\
Age $>75$ years & 4 \\
Anemia & 3 \\
Diabetes & 3 \\
Contrast-media volume & 1 per $100 \mathrm{ml}$ \\
SCr $>1.5 \mathrm{mg} / \mathrm{dl}(>132.6 \mu \mathrm{mol} / \mathrm{l})$ & 4 \\
or & 2 for $40-60$ \\
eGFR $<60 \mathrm{ml} / \mathrm{min}$ per $1.73 \mathrm{~m}^{2}$ & 4 for $20-39$ \\
& 6 for $<20$ \\
\hline
\end{tabular}

Low risk: cumulative score $<5$; high risk: cumulative score $>$ 16.Mehran R, AymongED,Nikolsky E, et al. A simple risk score for prediction of contrast-induced nephropathy after percutaneous coronary intervention. Development and initial validation.J Am CollCardiol.2004; 44:1393-1399.

Inall procedures, iohexol(Omnipaque $\left.{ }^{\circledR}\right)$ which is a water soluble low-osmolar contrast media has been used. A contrast volume of $3-5 / \mathrm{kg}$ body weight used and any volume larger regarded as an extra dose. ${ }^{1}$ Post procedure serum creatinine level had been checked on regular intervals; at 48 hours and on the seventh day. For those who fulfill the CNI-AKI definition criteria, renal function tests were followed by repeated measurements of Scr level on weekly bases for three months. A rise in S.Crlevel of greater than 25 percent above the baseline or an absolute rise of greater than $0.5 \mathrm{mg} / \mathrm{dl}$ considered abnormal. We didn't study the effect of pharmacological and non pharmacological interventions to prevent or to treat CIN-AKI.

\section{Statistical analysis:}

Data were tabulated and arrange by using (SPSS 14), NO, \% and mean \pm SD used to summarize data. Multivariate analysis was used to differentiate between independent and dependent factors. Association between variables were measured by chisquare test, difference between variables measured by t-test and ANOVA test $\mathrm{P}$ value $<0.05$ considered significant.

\section{Results:}

The mean age for the study group was $62 \pm 16.9$. Males were the predominant gender in the study $78(77 \%)$, while females were only $23(22.5 \%)$. Twenty three patients $(22.7 \%)$ were having diabetes mellitus. Only 13 patients $(12.7 \%)$ were having apre- 
existing renal impairment. Their mean baseline eGFR was 41 $\mathrm{ml} / \mathrm{min}$.

Ten patients $(9.9 \%)$ were high risk according to Mehran risk score. The contrast volume ranged from 50 to $500 \mathrm{ml}$ with mean of $301 \pm 145.34 \mathrm{ml}$. Thirty two patients $(31.6 \%)$ developed CIN by the definition within 48 hours of the procedure with the mean serum creatinine of $1.8 \mathrm{mg} / \mathrm{dl}$. The number of patients who developed renal impairment at weeks $1,2,3$, and 4 were $23(22.8 \%), 17(16.8 \%), 13(12.8 \%)$, and $7(6.8 \%)$ respectively. Those seven with persistently elevated serum creatinine at week 4 continued to have such impaired renal function up to three months of follow up.Twenty one patients (20.7\%) received extradoses of contrast. Factors that significantly associated with CIN were diabetes mellitus $(17 / 73.9 \%$, p value $<0.001)$, preexisting renal impairment $(13 / 100 \%$,p value $<0.0001)$, contrast volume $(16 / 84.2 \%, \mathrm{p}$ value $<0.0001)$. Other factors with significant associations are hypertension $(19 / 65.8 \%, p$ value $<0.001$ ), age ( $\mathrm{p}$ value $<0.025$ ), and myocardial infarction $(12 / 64.7 \%$, p value $<0.05)$. Multivariate analysis model identified that pre existing renal impairment ( $p$ value $<0.0001$ ), extra dose of contrast ( $p$ value $<0.0001$ ), DM ( $p$ value $<0.001$ ) , and (hypertension $\mathrm{p}$ value $<0.003$ ) were independent risk factors for the development and delayed recovery of Contrast induced AKI.

All seven patients who persistently had elevated serum creatinine at one month were diabetics, with baseline renal impairment and received extradoses of contrast (100\%). Table(2) shows the baseline characteristics of the study group.

Table (2) The Baseline Characteristics of the study group

\begin{tabular}{cc}
\hline Variables & Numbers \% \\
\hline Gender & $78(77.7)$ \\
Male & $23(22.3)$ \\
Female & $19-75(62 \pm 16.9)$ \\
\hline Age ( yr) & $19-25(22.6 \pm 1)$ \\
\hline BMI & $13(12.7)$ \\
\hline Pre-existing renal status & $23(22.7)$ \\
\hline DM & $30(29.7)$ \\
\hline HT & $32(31.7)$ \\
\hline MI & $27(26.7)$ \\
\hline Heart failures & $2(1.8)$ \\
\hline Shock/ IABP & 1 \\
\hline IABP & $50-700$
\end{tabular}

Table(3) discloses the distribution of the study group according to the independent risk factors by multivariate analysis.

Table (3) Distribution of the study group according to the independent risk factors by multivariate analysis.

\begin{tabular}{cccccccc}
\hline Risk factors & Pre test & Week 1 & Week 2 & Week 3 & Week 4 & 3 months & P - value \\
\hline Preexisting renal Impairment & $13(12.9)$ & $13(100.0)$ & $11(84.0)$ & $9(81.8)$ & $7(77.8)$ & $3(42.0)$ & 0.0001 \\
\hline Diabetes Mellitus & $23(22.5)$ & $17(73.9)$ & $13(68.4)$ & $\mathbf{8 ( 6 1 . 5 )}$ & $\mathbf{6 ( 6 0 . 0 )}$ & $4(66.7)$ & 0.001 \\
\hline Hypertension & $30(29.7)$ & $20(66.7)$ & $17(85.0)$ & $12(70.6)$ & $9(75.0)$ & $6(66.7)$ & 0.0001 \\
\hline Extra doses of contrast in $\mathrm{ml}$ & & $16(76.2)$ & $14(87.5)$ & $10(76.9)$ & $\mathbf{5 ( 5 5 . 6 )}$ & $\mathbf{5 ( 1 0 0 . 0 )}$ & $\mathbf{0 . 0 0 0 1}$ \\
\hline
\end{tabular}

\section{Discussion:}

Contrast-induced nephropathy (CIN) is a common, serious problem in the interventional cardiology practice that is associated with increased morbidity, mortality, and healthcare cost. This study is consistent with the international data of the incidence and risk factors of CIN.Here it's imperative to say that angiography in this study was for diagnostic and interventional purposes and for coronary and peripheral circulations. This is just to clarify the heterogeneous nature of the study sample and this probably had its impact on result in some way. There is no consensus on the definition of CIN used by the society of urogenital radiology and this add to the difficulty in designing studies on the incidence of CIN and Contrast associated AKI and this can explain the wide variation of incidence from $6 \%$ to $35 \%$ in many data. This is probably related to the cut off percentage of increment in serum creatinine or to the time of measurement( 48 hour vs. 72 hours or later ). Even the rate of day to day variation of serum creatinine in hospitalized patients has its effect on such definition and detection of CIN. In addition the background fluctuation of kidney function in patients receiving iodinated contrast has its impact on this definition. ${ }^{17,18}$ Validated risk-prediction models using patient and procedural risk factors to assess for CI-AKI have been developed for patients undergoing percutaneous coronary intervention like the Mehran risk model. The overall occurrence of CI-AKI in the development set of the score was $13.1 \%$ (range $7.5 \%$ to $57.3 \%$ for a low $[<5]$ and high $[>16]$ risk score, respectively); the rate of CI-AKI increased exponentially with increasing risk score. ${ }^{9,19}$ Diabetes Mellitus) DM (has significant effects on renal functionafter exposure to contrast mediaand this is consistent with the result of Parfery and Griffiths with a conclusion that even with normal renal function there is a little risk of clinically important nephrotoxicity attributable to contrast. ${ }^{20}$ Pre-existing renal impairment is a strong predictor 
for post catheterization renal impairment and there was strong association between pre-existence renal impairment and renal status one week after percutaneous coronary. This was consistent with the data from Rihal and Textor in their study. ${ }^{21,22}$ Moore et al. found a highly significant relationship $(p<0.001)$ between an increasing baseline level of serum creatinine and the frequency of nephrotoxicity (varying from $2 \%$ in those with baseline creatinine of $<1.5 \mathrm{mg} / \mathrm{dL}$ to $20 \%$ in those with levels of $>2.5 \mathrm{mg} / \mathrm{dL}$ ). ${ }^{23} \mathrm{In}$ this study hypertension had a significant effects on renal function and this is similar to that found by Mehran and Nikolsky et-al, 2004.The duration of HT had no significant effects ( $\mathrm{p}$ value <0.005)on the incidence while the effect of antihypertensive medications not studied with the predefined withdrawal of ACEIs and diuretics although their use may be of no effectaccording to some data like that of Goldenberg in $2005 .{ }^{9}$

In our study there was two patients with a systolic blood pressure of less than $100 \mathrm{~mm} \mathrm{hg}$, one presented acutely with cardioogenic shock and the other was on IABP. Both developed CIN and AKI. It's well known that hypotension and hypovolemia will augment the injurious effect of contrast on renal tubules but it is difficult to extrapolate data because of small sample. ${ }^{9}$ The volume of contrast was significantly associated with the risk of CIN . This was in accordance with the study of Mehran and colleagues.In this study the dose was based according to the worldwide standard practice of using contrast by $3-5 \mathrm{ml} / \mathrm{kg}$ body weight although the best practiceis to base thedose according to the renal function which can minimize contrast dose in patients with renal dysfunction and the resulting planned contrast volume restricted to less than thrice and preferablytwice the calculated creatinine clearance $\mathrm{CCC}$ might be valuable in reducing the risk of CIN. In another study a contrast media volume (CMV)/eGFR ratio > 5.35 was a significant and independent predictor of CIN in patients with CKD. ${ }^{9,24,25}$ CI-AKI is associated with an increased risk of mortality, cardiovascular events, renal failure, and prolonged hospitalization. However, the association between CI-AKI and mortality is strongly confounded by baseline clinical characteristics that simultaneously predispose to both kidney injury, delayed recovery, future risk of CKD/ESRD and mortality. The main baseline characteristics that confound such risk are diabetes mellitus and a preexisting impaired renal function as in this study. ${ }^{26}$ Despite all the standard prevention protocols seven patients developed and continued to have impaired renal function at one month after the procedure and all of them had coexistence of all these three risk factors.

\section{Conclusion:}

In conclusion, the risk of delayed renal recovery after contrast cannot be overlooked. Better attention on renal based contrast dose is mandatory. Larger longitudinal studies with ESRD risk and survival as the main outcome in relation to the risk factors will be certainly helpful.

\section{Authors contributions:}

Hilal Al-Saffar: The supervisor; Design the study, discusses results, and contribute in writing the manuscript.

Adel J Yassin: Design the study, collect the sample, calculate the results and write the draft of the manuscript.

Ala Ali: Revise the design of the study, critically review the draft of the manuscript, and discuss the renal part of the study.

\section{References:}

1. McCullough PA. Contrast induced acute kidney injury. J Am CollCardiol. 2008;51(15):1419-1428.

2. Majumdar SR, Kjellstrand CM, Tymchak WJ, HervasMalo M, Taylor DA, Teo KK. Forced euvolemic dieresis with mannitol and furesomide for prevention of contrast-induced nephropathy in patients with CKD undergoing coronary angiography: a randomized controlled trial. Am J Kidney Dis. 2009;54(4): 602-609.

3. Burns KE, Chu MW, Novick RJ, et al. Perioperative $N$-acetylcysteine to prevent renal dysfunction in high-risk patients undergoing CABG surgery: a randomized controlled trial. JAMA 2005; 294: 342-350

4. Baumgarten DA, Ellis JH. Contrast-induced nephropathy: contrast material not required? AJR Am J Roentgenol2008; 191: 383-386.

5. Rudnick MR, Goldfarb S, Tumlin J. Contrast-induced nephropathy: is the picture any clearer? Clin J Am SocNephrol2008; 3: 261-262.

6. Pakfetrat M, Nikoo MH, Malekmakan L, et al. Risk factors for contrast-related acute kidney injury according to risk, injury, failure, loss, and end-stage criteria in patients with coronary interventions. Iran J Kidney Dis. 2010; 4(2):116122.

7. Persson PB, Hansell P, Liss P. Pathophysiology of contrast medium-induced nephropathy. IntSocNephrol. 2005;68:1422.

8. Anna Kagan, and David Sheikh-Hamad. Contrastinduced Kidney Injury: Focus on Modifiable Risk Factors and Prophylactic Strategies Clin. Cardiol. 33, 2, 62-66 (2010).

9. Mehran R, Aymong ED, Nikolsky E, etal.a simple risk score for prediction of contrast induced nephropathy after percutaneous coronary intervention: development and initial validation. J Am CollCardiol; 2004: 44:1393-9.

10. Richard J. Solomon, Roxana Mehran,et al. ContrastInduced Nephropathy and Long-Term Adverse Events: Cause and Effect? Clin J Am SocNephrol. Jul 2009; 4(7): 11621169.

11. William F. Finn. The clinical and renal consequences of contrast-induced nephropathy. Nephrol Dial Transplant 
(2006) 21 [Suppl 1]: i2-i10

12. McCullough PA, Wolyn R, Rocher LL, Levin RN, $O$ 'Neill WW.Acute renal failure after coronary intervention: incidence, risk factors, and relationship to mortality. Am J Med 1997;103: 368-375

13. Sadeghi HM et al. Impact of renal insufficiency in patients undergoing primary angioplasty for acute myocardial infarction.Circulation 2003; 108: 2769-2775

14. Gruberg $L$ et al. The prognostic implications of furtherrenalfunction deterioration within 48 h of interventional coronary procedures in patients with pre-existent chronic renal insufficiency. J Am CollCardiol 2000; 36:1542-1548

15. Wi J et al. Prediction of Contrast-Induced Nephropathy With Persistent Renal Dysfunction and Adverse Long-term Outcomes in Patients With Acute Myocardial Infarction Using the Mehran Risk Score.ClinCardiol. 2013 Jan;36(1):46-53.

16. Donald S. Baim Daniel I. Simona. (2006). Complications and the Optimal Use of Adjunctive Pharmacology. In: DONALD S. BAIM, Grossman's Cardiac Catheterization, Angiography, and Intervention, $7^{\text {th }}$ edition. 7th ed. Philadelphia: LWW. 53.

17. Thomsen HS, Morcos SK. Contrast media and the kidney: European Society of Urogenital Radiology (ESUR) guidelines. Br J Radiol2003; 76: 513-518.

18. Bruce RJ, Djamali A, Shinki K, et al. Background fluctuation of kidney function versus contrast-induced nephrotoxicity. AJR Am J Roentgenol 2009; 192: 711-718.

19. Brown JR, DeVries JT, Piper WD, et al. Serious renal dysfunction after percutaneous coronary interventions can be predicted. Am Heart J 2008; 155: 260-266.IVSL
20. Perfery PS, Griffiths SM, Barret BJ, etal.contrast material induced nepheropathy in patientswiyh diabetes mellitus, renal insufficiency, or both. A prospective control study. N Engle J Med; 1989;320:143-9.

21. Rihal CS, Textor SC, et al. incidence and prognostic importance of acute renal failure after percutaneous coronary intervention. Circulation 2002;105:2259-64.

22. Goldenberg I, Matetzky S. Nephropathy induced by contrast media: pathogenesis, risk factors and preventive strategies. CMAJ. 2005; 24:172:1461-71.

23. Moore RD, Steinberg EP, Power NR, et al. Nephrotoxicity of high-osmolality versus low-osmolality contrast media: randomized clinical trial. Radiology1992; 182:649-655

24. Hitinder S. Gurm, et al. Renal Function-Based Contrast Dosing to Define Safe Limits of Radiographic Contrast Media in Patients Undergoing Percutaneous Coronary Interventions. JACC 2011; 58:907-914.IVSL

25. SoichiroEbisawa, et al. The ratio of contrast media volume to estimated glomerular filtration rate as a new marker to prevent contrast induced nephropathy in patients with percutaneous coronary interventions. JACC. 2011; 14 : E1905.

26. James MT, Samuel SM, Manning MA, et al. Contrast induced acute kidney injury and risk of adverse clinical outcomes after coronary angiography: a systematic review and meta-analysis.CircCardiovascInterv. 2013 ;6:37-43. 